\title{
ALTITUDE ESTIMATION OF MANEUVERING TARGETS IN MIMO OVER-THE-HORIZON RADAR
}

\author{
Yimin D. Zhang ${ }^{\dagger}$, Moeness G. Amin ${ }^{\dagger}$, and Braham Himed ${ }^{\ddagger}$ \\ $\dagger$ Center for Advanced Communications, Villanova University, Villanova, PA 19085, USA \\ $\ddagger$ Air Force Research Laboratory, AFRL/RYMD, Dayton, OH 45433, USA
}

\begin{abstract}
Target altitude estimation is an important problem in overthe-horizon radar (OTHR) systems for target tracking and classification. Narrow signal bandwidth and rich propagation environment in OTHR render this problem difficult and challenging. In this paper, we develop a novel technique that allows accurate altitude estimation for a maneuvering target during its ascending or descending flight. The proposed technique considers a multiple-input multiple-output (MIMO) OTHR platform and utilizes the difference in the Doppler signatures generated in a micro-multipath propagation environment. The separability of the multi-component Doppler signatures in the time-frequency domain is used to individually estimate the elevation angles of these paths, which are otherwise infeasible to obtain, due to their small angular separation. The estimated elevation angle information is then used to achieve a reliable estimate of the target altitude.
\end{abstract}

\section{INTRODUCTION}

Over-the-horizon radar (OTHR) systems are critical for national defense and other missions because of their wide-area surveillance capabilities to cover long ranges that are well beyond the limit of conventional line-of-sight (LOS) radars [1]-[2]. Skywave OTHR systems exploit the reflective and refractive nature of high-frequency (HF) radiowave propagation through the ionosphere to cover a surveillance area up to thousands of kilometers.

Because of the narrow signal bandwidth, the range resolution of an OTHR system is usually limited. In particular, target altitude information is difficult to obtain and remains a challenging problem in OTHR. This information, even in the order of a thousand meters, can be very helpful in identifying the type of aircraft and determining the flight levels of commercial aircrafts. Although there have been various attempts to estimate the absolute or relative altitude information $[3,4,5]$, absolute target altitude estimation in OTHR has not received proper attention.

A practical and tangible method for providing the altitude information is based on micro-multipath model that makes use of multipath returns due to the ocean or ground reflections local to the target [3]. The micro-multipath

The work of Y. D. Zhang and M. G. Amin is supported in part by a subcontract with Dynetics, Inc. for research sponsored by the Air Force Research Laboratory (AFRL) under Contract FA865008-D-1303. propagation is illustrated in Fig. 1 with a simplified flatearth ionosphere model [4]. Changes in the target altitude will alter the distance of the direct path and the multipath, generating Doppler variations, with opposite signs, around the nominal Doppler signature. These propagation paths of the emitted/received signals associate themselves with different non-linear time-frequency trajectories, each correspond to a Doppler signature of the target along a propagation path.

It is shown in [4] that high-resolution time-frequency analysis of the Doppler signatures can provide resolution of the Doppler signatures, enabling fast and robust tracking of the altitude changes. However, the non-linearity and close separation of the Doppler structures still render analyses of such signals difficult and challenging $[4,5]$. Recent works $[6$, 7] have developed new approaches for accurate parametric estimations of time-varying multi-component signals with closely separated Doppler signatures encountered in OTHR systems.

In this paper, we utilize successful separation of the multi-component Doppler signatures due to micro-multipath propagation in a MIMO OTHR platform to estimate the absolute altitude of a maneuvering target during its ascending or descending flight. It is noted that the separability of the multiple signal components in the time-frequency domain has several advantages, including estimation of the directions of closely spaced targets that otherwise are difficult to resolve based only on spatial processing [8].

The following notations are used in this paper. A lower (upper) case bold letter denotes a vector (matrix). E[.] represents the statistical mean operation. $(\cdot)^{*},(\cdot)^{T}$ and $(\cdot)^{H}$ respectively denote complex conjugation, transpose, and conjugate transpose (Hermitian) operations. $\mathbf{I}_{n}$ expresses the $n \times n$ identity matrix. $\operatorname{Diag}(\mathbf{x})$ denotes a diagonal matrix using the elements of $\mathbf{x}$ as its diagonal elements. In addition, $\mathbb{C}^{N \times M}$ denotes the complete set of $N \times M$ complex entries.

\section{SIGNAL MODEL}

\subsection{OTHR Micro-Multipath Model}

Consider an OTHR system using the flat-earth model as illustrated in Fig. 1. In practice, the transmit and receive array are separated by a distance, which is much smaller than the range, to mitigate power leakage. We assume that the difference of the range due to the array positions is negligible. Thus, the slow-time representation of the received 


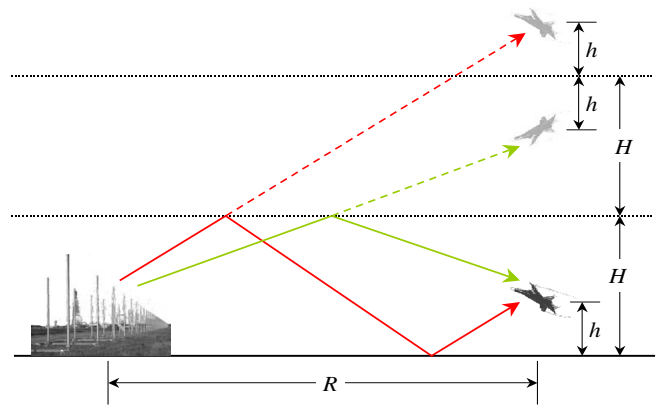

Figure 1: Micro-multipath propagation in OTHR.

signal is expressed as

$$
y(t)=\sigma_{s} \gamma(t)+z(t)=\sigma_{s} \rho e^{j \phi(t)}+z(t),
$$

where $t$ is the slow-time index, $\sigma_{s}^{2}$ is the power of the transmitted signal waveform, $\gamma(t)=\rho e^{j \phi(t)}$ is the complex reflection coefficient of the target, and $z(t)$ is noise.

Denote $l_{1}$ and $l_{2}$ as the propagation distances of the two paths observed at the arrays, respectively. Then, the combination of the direct path and the multipath in both forward and return links yields the following three combinations of the two-way propagation: path I $\left(l_{1}: l_{1}\right)$, path II $\left(l_{2}: l_{2}\right)$, path III $\left(l_{1}: l_{2}\right.$ and $\left.l_{2}: l_{1}\right)$. Therefore, the received signal consists of the following three components:

$$
y(t)=\sum_{i=1}^{3} \sigma_{s} \gamma_{i}(t)+z(t)=\sum_{i=1}^{3} \sigma_{s} \rho_{i} e^{j \phi_{i}(t)}+z(t),
$$

where the phase term is related to the propagation paths by $\phi_{i}(t)=-2 \pi f_{c}\left(l_{t, k_{t}(i)}+l_{r, k_{r}(i)}\right) / c$, where $f_{c}$ is the carrier frequency, $c$ is the propagation velocity, and $l_{t, k_{t}(i)}$ and $l_{r, k_{r}(i)}$ respectively denote the slant range of the forward and return links associated with path $i$.

The slant ranges $l_{1}$ and $l_{2}$ can be expressed in terms of the ground range distance $R$, the ionosphere altitude $H$, and the target altitude $h$, as

$$
l_{1}=\left(R^{2}+(2 H-h)^{2}\right)^{1 / 2}, \quad l_{2}=\left(R^{2}+(2 H+h)^{2}\right)^{1 / 2} .
$$

Equation (3) is used for numerical evaluations of the slant ranges in this paper. Nevertheless, for illustrative purpose, we also use the following approximations, which hold true when $R \gg H \gg h$, to reveal a clear relationship between the slant ranges and the other parameters:

$$
l_{1} \approx R+\frac{2 H^{2}-2 H h}{R}, \quad l_{2} \approx R+\frac{2 H^{2}+2 H h}{R} .
$$

\subsection{MIMO Radar Signal Model}

We use a bistatic MIMO radar model for the direction finding of the OTHR system, which operates in a pseudomonostatic mode. The MIMO radar consists of $N_{t}$ closely spaced transmit antennas and $N_{r}$ closely spaced receive antennas. Denote $\mathbf{S}$ as the narrowband waveform matrix which is assumed to be orthonormal in the fast-time domain, i.e., $\mathbf{S S}^{H}=\mathbf{I}_{N_{t}}$.
Consider the $L=3$ multipath components as described earlier, which correspond to two direction-of-departure (DOD) angles $\theta_{t, k_{t}(i)}$ and two direction-of-arrival (DOA) angles $\theta_{r, k_{r}(i)}, i=1, \cdots, L$. For simplicity and without loss of generality, we only consider the elevation angle $\theta$ which is closely related to the target altitude. Then, the signal data received at the receive array corresponding to the range cell is expressed in the slow-time domain as the following $N_{r} \times T$ complex matrix

$$
\mathbf{X}(t)=\mathbf{A}_{r} \boldsymbol{\Gamma}(t) \mathbf{A}_{t}^{H} \mathbf{S}+\mathbf{N}(t),
$$

where $\mathbf{A}_{t}=\left[\mathbf{a}_{t}\left(\theta_{t, k_{t}(1)}\right), \cdots, \mathbf{a}_{t}\left(\theta_{t, k_{t}(L)}\right)\right] \quad$ and $\quad \mathbf{A}_{r}=$ $\left[\mathbf{a}_{r}\left(\theta_{r, k_{r}(1)}\right), \cdots, \mathbf{a}_{r}\left(\theta_{r, k_{r}(L)}\right)\right]$, with $\mathbf{a}_{t}\left(\theta_{t, k_{t}(i)}\right) \in \mathbb{C}^{N_{r} \times 1}$ and $\mathbf{a}_{r}\left(\theta_{r, k_{r}(i)}\right) \in \mathbb{C}^{N_{t} \times 1}$ respectively denoting the transmit and receive steering vectors corresponding to the $i$ th two-way path. In addition, $\boldsymbol{\Gamma}(t)=\operatorname{Diag}\left[\gamma_{1}(t), \cdots, \gamma_{L}(t)\right]$. Moreover, we assume that the target has a high nominal Doppler frequency that clearly distinguish itself from the clutter region. The signal located in the clutter region may be excluded for direction finding. Thus, $\mathbf{N}(t) \in \mathbb{C}^{N_{r} \times T}$ is additive noise matrix, whose elements are assumed to be independent and identically distributed (i.i.d.) complex Gaussian random variables with zero mean and variance $\sigma_{n}^{2}$. To justify expression (5), it is assumed that the steering vectors remain unchanged during the entire slow-time processing period, which is often the case for far-field targets.

By post-multiplying (5) by $\mathbf{S}^{H}$, we obtain $\mathbf{Y}(t) \in \mathbb{C}^{N_{t} \times N_{r}}$ as

$$
\mathbf{Y}(t)=\mathbf{A}_{r} \boldsymbol{\Gamma}(t) \mathbf{A}_{t}^{H}+\mathbf{Z}(t),
$$

where $\mathbf{Z}(t)=\mathbf{N}(t) \mathbf{S}^{H}$.

\subsection{Target Elevation Angle and Doppler Signatures}

The elevation DOD/DOA of the two paths, path I and path II, can be expressed as

$$
\theta_{1}=\tan ^{-1}\left(\frac{2 H-h}{R}\right), \quad \theta_{2}=\tan ^{-1}\left(\frac{2 H+h}{R}\right) .
$$

The angular separation between paths I and II is usually very small and simultaneous estimation of these angles using high-resolution direction finding techniques would thus be infeasible.

The maneuvering of a target, in general, consists of horizontal and elevation movements. For our purpose, we consider the Doppler frequency characteristics of the target maneuvering in the two different dimensions. For a maneuvering target, we consider that both $R$ and $h$ are time-varying, whereas the height of ionosphere, $H$, is considered constant over the processing time.

The Doppler frequencies of the three different paths are then obtained as

$$
\begin{aligned}
& f_{D, 1}(t)=\frac{2 f_{c}}{c} \frac{d l_{1}(t)}{d t} \approx \frac{2 f_{c}}{c} K(t) v_{R}(t)-\frac{4 f_{c} H}{R(t) c} v_{c}(t), \\
& f_{D, 2}(t)=\frac{2 f_{c}}{c} \frac{d l_{2}(t)}{d t} \approx \frac{2 f_{c}}{c} K(t) v_{R}(t)+\frac{4 f_{c} H}{R(t) c} v_{c}(t), \\
& f_{D, 3}(t)=f_{D, 4}(t)=\frac{f_{c}}{c} \frac{d l_{1}(t)+d l_{2}(t)}{d t} \approx \frac{2 f_{c}}{c} K(t) v_{R}(t),
\end{aligned}
$$


where $K(t)=1-2 H^{2} / R^{2}(t), v_{R}(t)=d R(t) / d t$ is the target velocity in the range direction toward the radar, and $v_{c}(t)=$ $d h(t) / d t$ is the ascending velocity of the target.

From the above discussion, it is evident that, while the dominant Doppler component $\bar{f}_{D}=2 f_{c} K(t) v_{R}(t) / c$ is shared by all the three paths and reveals the target velocity in the range direction, the small Doppler difference between the paths, $\tilde{f}_{D}= \pm 4 f_{c} H v_{c}(t) /[R(t) c]$, is a function of $h(t)$. The Doppler frequency difference allows separation of the multi-component Doppler signatures so that only a single component is selected for DOD and DOA estimations.

\section{TARGET ALTITUDE ESTIMATION}

Several techniques have been developed to discriminate targets/sources based on time-frequency signatures (see e.g., $[6,9])$. In this paper, we assume that perfect Doppler estimation is achieved for all the multipath components.

Rewrite (6) as

$$
\mathbf{Y}(t)=\sum_{i=1}^{L} \sigma_{s} \rho_{i}(t) \mathbf{A}_{i} e^{j \phi_{i}(t)}+\mathbf{Z}(t),
$$

where $\mathbf{A}_{i}=\mathbf{a}_{r}\left(\theta_{r, k_{r}(i)}\right) \mathbf{a}_{t}^{H}\left(\theta_{t, k_{t}(i)}\right)$. Stationarizing $\mathbf{Y}(t)$ using the estimated phase $\psi_{k}(t), k=1,2$, which is assumed to be perfectly estimated, yields

$$
\begin{aligned}
& \mathbf{Y}(t) e^{-j \psi_{k}(t)}=\sigma_{s} \rho_{k}(t) \mathbf{A}_{k} \\
& \quad+\sum_{i=1, i \neq k}^{L} \sigma_{s} \rho_{i}(t) \mathbf{A}_{i} e^{j \phi_{i}(t)-j \psi_{k}(t)}+\mathbf{Z}(t) e^{-j \psi_{k}(t)}
\end{aligned}
$$

Therefore, the desired signal component corresponding to the $k$ th path becomes a stationary DC signal, whereas the other multipath components will have non-zero frequencies, provided that $f_{D, i} \neq f_{D, k}$ for $i \neq k$. As such, averaging the above result over the observation slow-time amounts to low-pass filtering the signal, which, ideally, will capture the desired component and filter out the multipath components represented by the second term in the right-hand side of the above expression. Further, the averaging operation, given below, reduces the noise power proportionally to the coherent integral time. That is,

$$
\overline{\mathbf{Y}}^{[k]}=\frac{1}{T_{0}} \sum_{t=0}^{T_{0}-1} \mathbf{Y}(t) e^{-j \psi_{k}(t)}=\sigma_{s} \rho_{k}(t) \mathbf{A}_{k}+\overline{\mathbf{Z}}^{[k]},
$$

where $T_{0}$ is the total number of slow-time samples used for the operation, and $\overline{\mathbf{Z}}^{[k]}=\left(1 / T_{0}\right) \sum_{t=0}^{T_{0}-1} \mathbf{Z}(t) e^{-j \psi_{k}(t)}$ is the noise component after filtering. All the spatial signature of the $k$ th path is carried in $\mathbf{A}_{k}$ in the above expression. With the other multipath components removed or highly mitigated, and the noise reduced, accurate DOD and DOA estimation of the $k$ th path becomes possible. This procedure is repeated for $k=1$ and 2. For better isolation and filtering, a proper window (e.g., Hamming window) is used to reduce the sidelobe leakage of undesired signal components.

The joint DOD/DOA estimation in a bistatic MIMO radar system, in general, may involve a two-dimensional search. A number of techniques have been developed to simplify the two-dimensional search into decoupled onedimensional direction finding problem and perform pairing of the DOD and DOA information when multiple targets are present (e.g., $[10,11,12])$. In the underlying problem, the DOD/DOA of only a single path is estimated each time. Therefore, no pairing procedure is required. In this paper, we use the MUSIC-based technique, where the DOD and DOA are estimated from $\overline{\mathbf{Y}}^{[k]}$.

Once the elevation angle of paths I and II are estimated as $\theta_{1}$ and $\theta_{2}$, we can readily obtain the nominal altitude of the target from Fig. 1 using

$$
\hat{h}=\frac{1}{2}\left[l_{2} \sin \left(\theta_{2}\right)-l_{1} \sin \left(\theta_{1}\right)\right] .
$$

The above operation can be performed for both DOD and DOA estimates. Notice that the actual target altitude is time-varying, and the above estimate reflects its mean value during the observation period. The instantaneous target altitude can be obtained by incorporating its relative altitude changes addressed for example in $[4,5]$.

\section{SIMULATION RESULTS}

As an example, we consider a maneuvering aircraft which makes a $360^{\circ}$ circular turn of radius $5 \mathrm{~km}$ in approximately $T=179.5$ seconds to descend by approximately 2,250 meters. The centers of the transmit and receive arrays are set as the coordinate origins. The other key simulation parameters are listed in Table I. All the multipath signals are considered to fall within the same range cell. The aircraft maintains a horizontal velocity of $175 \mathrm{~m} / \mathrm{s}(630 \mathrm{~km} / \mathrm{hr})$. Its elevation velocity varies sinusoidally, and the corresponding target altitude is expressed as

$$
h(t)=h(0)-\frac{v_{c, \max } T_{0}}{\pi}\left[1-\cos \left(\frac{\pi t}{T_{0}}\right)\right] .
$$

Table 1: Key Parameters

\begin{tabular}{lcr}
\hline Parameter & Notation & Value \\
\hline initial range & $R(0)$ & $1,500 \mathrm{~km}$ \\
ionosphere height & $H$ & $160 \mathrm{~km}$ \\
aircraft initial height & $h(0)$ & $10,000 \mathrm{~m}$ \\
horizonal target velocity & $v_{R, \max }$ & $175 \mathrm{~m} / \mathrm{s}$ \\
maximum descending velocity & $v_{c, \max }$ & $19.68 \mathrm{~m} / \mathrm{s}$ \\
carrier frequency & $f_{c}$ & $16 \mathrm{MHz}$ \\
waveform repetition frequency & $f_{s}$ & $40 \mathrm{~Hz}$ \\
\hline
\end{tabular}

The actual Doppler signatures obtained from (3) are depicted in Fig. 2(a), and those obtained from the data, after the path I component is made stationary using its exact Doppler signature, are shown in Fig. 2(b). The maximum value of the one-side Doppler difference is $0.448 \mathrm{~Hz}$. The first and last 20 seconds are excluded from the DOD/DOA estimation because of the close Doppler signatures between the different multipath components. A Hamming window of length 5601, which spans the entire selected time period of approximately 140 seconds, is used to mitigate the sidelobe leakage.

For simplicity and without loss of generality, we consider small-size arrays that use 6 transmit antennas and 10 receive antennas. Both arrays are linear and the antennas are extended in the range direction. To achieve a large array aperture, the minimum redundancy array (MRA) configurations [13] are used at both the transmit and receive arrays, where the unit separation is one wavelength. 


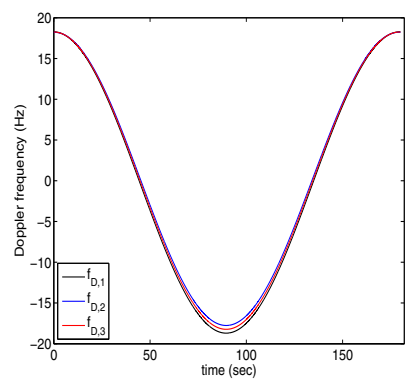

(a) before stationarization

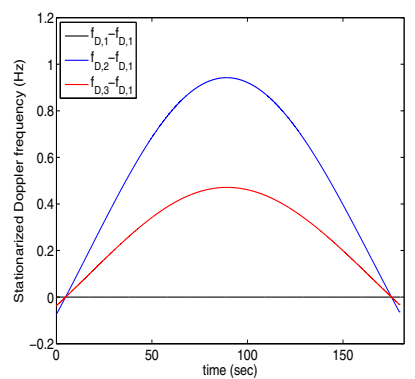

(b) after stationarization
Figure 2: Multipath Doppler signatures.

In Fig. 3, the MUSIC pseudo spatial spectra are shown for 10 independent estimates respectively obtained for DOD and DOA, where the input SNR is $-10 \mathrm{~dB}$. In Fig. 4, the root mean square error (RMSE) of the mean target altitude versus the input SNR is obtained by averaging over 100 independent trials. It is evident from these results that the elevations angles of the multipath components are clearly resolved for target altitude estimation. The DOA estimates are generally of better quality when compared with the DOD estimates because more sensors are used at the receiver. The RMSE of the mean altitude estimate is improved by an order of magnitude as the input SNR is increased by $20 \mathrm{~dB}$. Note that a $1 \mathrm{~km}$ RMSE is achieved for the small array configurations even when the input SNR is below $-20 \mathrm{~dB}$.



(a) DOD

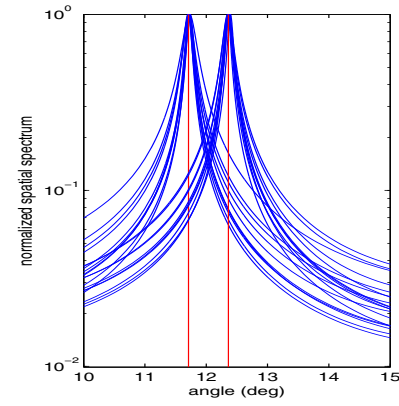

(b) DOA
Figure 3: DOD and DOA estimates.

\section{CONCLUSION}

We have developed a novel technique for the estimation of a maneuvering target altitude in a MIMO OTHR system. By utilizing the difference between the multi-component Doppler signatures due to micro-multipaths, the proposed technique is based on individual estimation of the DOD and DOA information. Further, the knowledge of the nonlinear Doppler signatures, which can be estimated by using recently developed time-frequency analysis techniques, enables them to become stationary for achieving SNR enhancements through extended coherent integration times. Simulation results have shown that the proposed technique is very promising in a realistic low SNR environment.

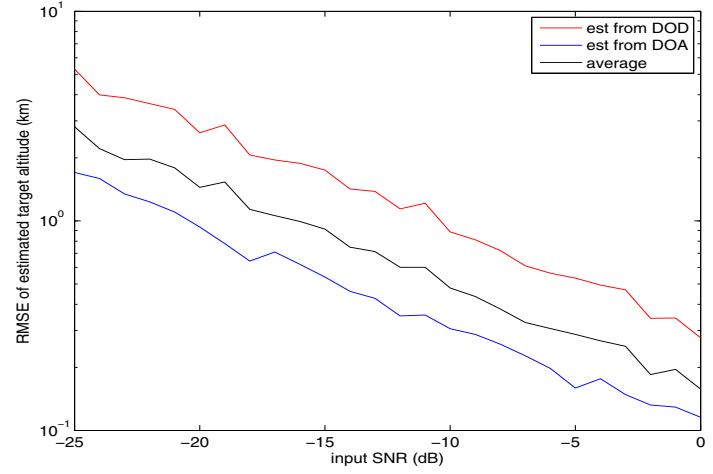

Figure 4: RMSE of the target altitude estimates for different input SNR.

\section{REFERENCES}

[1] J. M. Headrick and M. I. Skolnik, "Over-the-horizon radar in the HF band," Proc. IEEE, vol. 62, pp. 664-673, 1974.

[2] A. A. Kolosov, Over-the-Horizon Radar, Boston, MA: Artech House, 1987.

[3] R. H. Anderson, S. Kraut, and J. L. Krolik, "Robust altitude estimation for over-the-horizon radar using a statespace multipath fading model," IEEE Trans. Aerospace Electron. Syst., vol. 39, no. 1, pp. 192-201, Jan. 2003.

[4] Y. Zhang, M. G. Amin, and G. J. Frazer, "High-resolution time-frequency distributions for manoeuvring target detection in over-the-horizon radars," IEE Proc.-Radar Sonar Navig., vol. 150, no. 4, pp. 299-304, Aug. 2003.

[5] C. Ioana, M. G. Amin, Y. D. Zhang, and F. Ahmad, "Characterization of Doppler effects in the context of over-thehorizon radar," in Proc. IEEE Int. Radar Conf., Washington D.C., May 2010.

[6] C. Ioana, Y. D. Zhang, M. G. Amin, F. Ahmad, G. Frazer, and B. Himed, "Time-frequency characterization of micromultipath signals in over-the-horizon radar," in Proc. IEEE Int. Radar Conf., Atlanta, GA, May 2012.

[7] C. Ioana, Y. D. Zhang, M. G. Amin, F. Ahmad, and B. Himed, "Time-frequency analysis of multipath Doppler signatures of maneuvering targets," in Proc. IEEE Int. Conf. Acoust., Speech, and Signal Proc., Kyoto, Japan, March 2012.

[8] Y. Zhang, W. Mu, and M. G. Amin, "Subspace analysis of spatial time-frequency distribution matrices," IEEE Trans. Signal Proc., vol. 49, no. 4, pp. 747-759, April 2001.

[9] S. Barbarossa and O. Lemoine, "Analysis of nonlinear FM signals by pattern recognition of their time-frequency representation," IEEE Signal Proc. Lett., vol. 3, no. 4, pp. 112115, April 1996.

[10] M. Jin, G. Liao, and J. Li, "Joint DOD and DOA estimation for bistatic MIMO radar," Signal Processing, vol. 89, pp. 244-251, 2009.

[11] M. L. Bencheikh and Y. Wang, "Joint DOD-DOA estimation using combined ESPRIT-MUSIC approach in MIMO radar," Electronics Letters, vol. 46, no. 15, July 2010.

[12] Y. D. Zhang and M. G. Amin, "MIMO radar for direction finding with exploitation of time-frequency representations," in Proc. IEEE Int. Conf. Acoustics, Speech, and Signal Proc., Plague, Czech Republic, May 2011.

[13] A. T. Moffet, "Minimum-redundancy linear arrays," IEEE Trans. Antennas Propagat., vol. AP-16, pp. 172-175, March 1968. 